\title{
Mental Health Stigma and Discrimination in Ethiopia: Evidence Synthesis to Inform Stigma Reduction Interventions
}

Eshetu Girma ( $\square$ yaneteshetu@gmail.com )

Addis Ababa University https://orcid.org/0000-0002-9418-1775

Bezawit Ketema

Addis Ababa University School of Public Health

Tesfahun Mulatu

Addis Ababa University School of Public Health

Brandon A Kohrt

The George Washington University

Syed Shabab Wahid

The George Washington University Milken Institute of Public Health

Eva Heim

University of Zurich Department of Psychology: Universitat Zurich Psychologisches Institut

Petra C Gronholm

King's College London

Charlotte Hanlon

: Addis Ababa University College of Health Sciences

Graham Thomicroft

King's College London

Research

Keywords: Stigma, Mental health, Discrimination, Ethiopia

Posted Date: August 17th, 2021

DOl: https://doi.org/10.21203/rs.3.rs-789548/v1

License: @ (1) This work is licensed under a Creative Commons Attribution 4.0 International License. Read Full License 


\section{Abstract \\ Background}

People with mental illnesses are at an increased risk of experiencing human rights violations, stigma and discrimination. Even though mental health stigma and discrimination are universal, there appears to be a higher burden in low- and middle-income countries. Anti-stigma interventions need to be grounded in local evidence. The aim of this paper was to synthesize evidence on mental health stigma and discrimination in Ethiopia to inform the development of antistigma interventions.

\section{Methods}

This evidence synthesis was conducted as a part of formative work for the International Study of Discrimination and Stigma Outcomes (INDIGO) Partnership research program. Electronic searches were conducted using PubMed for scientific articles, and Google Search and Google Scholar were used for grey literature. Records fulfilling eligibility criteria were selected for the evidence synthesis. The findings were synthesized using a framework designed to capture features of mental health stigma to inform cultural adaptation of anti-stigma interventions.

\section{Results}

A total of 37 records (2 grey literature and 35 scientific articles) were included in the evidence synthesis. Some of these records were described more than once depending on themes of the synthesis. The records were synthesized under the themes of explanatory models of stigma (3 records on labels and 4 records on symptoms and causes), perceived and experienced forms of stigma (7 records on public stigma, 6 records on structural stigma, 2 records on courtesy stigma and 4 records on self-stigma), impact of stigma on help-seeking (6 records) and interventions to reduce stigma (12 records). Only two intervention studies assessed stigma reduction- one study showed reduced discrimination due to improved access to effective mental health care, whereas the other study did not find evidence on reduction of discrimination following a community-based rehabilitation intervention in combination with facility-based care.

\section{Conclusion}

There is widespread stigma and discrimination in Ethiopia which has contributed to under-utilization of available mental health services in the country. This should be addressed with contextually designed and effective stigma reduction interventions that engage stakeholders (service users, service providers, community representatives and service developers and policy makers) so that the United Nations universal health coverage goal for mental health can be achieved in Ethiopia.

\section{Introduction}

According to the Global Burden of Diseases, Injuries, and Risk Factors Study 2017, the percentage of global age-standardized Years Lived with Disability (YLD) attributed to mental disorders was $14 \%$, which has had comparably similar levels for nearly three consecutive decades. The study indicated that globally, more than one billion people live with mental disorders and/or substance use disorders. Of these nearly 163 million, 19 million and 175 million people live with major depressive disorder, schizophrenia and substance use disorder, respectively, with depressive disorder being one of the top three leading causes of YLDs in the world [1].

The World Health Organization (WHO) special initiative for mental health highlighted that nearly four out of five people with mental illness (including substance use and neurological disorders) do not receive good quality and affordable mental health care globally [2]. People with mental illnesses are prone to suffer violations of human rights and to experience different forms of stigma and discrimination [2-4]. Even though the problems of stigma and discrimination are known to be universal, their magnitude and severity vary across countries. Thus, low- and middle-income countries (LMICs) have a higher burden compared with high-income countries [5].

Stigma can be conceptualized as experiential (perceived stigma, endorsed stigma, anticipated stigma, received stigma and enacted stigma) or action oriented (public stigma, structural stigma, courtesy stigma, provider-based stigma, and self-stigma). The experiential stigma categories describe how stigma occurs, whereas action oriented stigma forms indicate who/what gives or receives the stigma [6, 7]. Stigma and discrimination against people with mental illnesses has been described as bringing more challenges to affected people than their primary problem of mental illness itself [5].

In Ethiopia the prevalence of schizophrenia, bipolar disorder and major depression is reported as $0.5 \%, 0.63 \%$ and $6.8 \%$, respectively [8, 9]. In a follow up study in a rural part of the country it was highlighted that people with severe mental disorders had a substantially increased risk of death, $13.2 \%$ died over a 10 year period, which was almost twice as high as the risk among the general population and amounted to 28 years of life lost per person [10]. Mortality was mostly due to physical health conditions, suggesting that reduced access to healthcare could have contributed, and was shown to be reduced when people had access to mental health care [10, 11]. The health metrics estimate for Ethiopia in 2017 showed that the YLDs for depression, anxiety, bipolar disorder and schizophrenia contributed an estimated $5.32 \%, 3.60 \%, 1.23 \%$, and $0.84 \%$ of total YLDs, respectively [9].

In low-income countries, like Ethiopia, mental disorders are not considered as life threatening, and have not been given due attention by policy makers and service providers [12]. To address this gap, Ethiopia has implemented scale up of mental health care through integration into primary health care and general medical service since 2012 [13] and planned to have these service in all districts by the end of 2020 [14]. 
Intervention approaches commonly used to reduce stigma against people with mental disorders are education, contact and protest [15]. The approach of education focuses on enhancing knowledge and awareness, providing factual information in order to reverse myths, wrongly held beliefs and negative attitudes. The approach of contact (also called social or inter-personal contact) can be direct or indirect and aims to enhance interaction and connection between people through sharing of lived experience, recovery stories and challenges so that the audience can overcome fear, build self-esteem and develop empathy. The approach of protest consists of advocacy and emphasizing issues of civil rights $[7,16]$. Of these approaches, the strategies known to be most effective are education and contact [7, 17-19].

It is very important to consider the variants of stigma and specific groups affected in order to design effective interventions targeting reduction of stigma and discrimination. This could be achieved by understanding the context of the community where the problem has occurred [7, 20, 21]. However, there is a lack of up-to-date reviews that synthesize evidence on mental health stigma and discrimination at the country level in Ethiopia.

The International Study of Discrimination and Stigma Outcomes (INDIGO) is a collaboration of research colleagues in over 40 countries worldwide committed to developing knowledge about mental-illness-related stigma and discrimination, both in terms of their origins and their eradication [22]. INDIGO is coordinated by the Centre for Global Mental Health, Institute of Psychiatry, Psychology and Neuroscience at King's College London. Since its inception in 2006, the Indigo Network has produced substantial evidence on the impact of stigma and discrimination [3,23-28] and has now moved its focus towards identifying methods to reduce stigma and discrimination $[5,19,29]$. A detailed description of the program is available on the INDIGO website (http://www.indigo-group.org).

This evidence synthesis was conducted as a part of the UK Medical Research Council-funded INDIGO Partnership research program which aims to develop and test new methods to reduce mental-health related stigma in China, Ethiopia, India, Nepal and Tunisia. The program is aimed at establishing a research collaboration that enables the development of effective, contextually adapted stigma and discrimination reduction interventions, and at carrying out activities to strengthen the scientific understanding of mechanisms of action of stigma processes against people with mental illness. As part of the formative work in the program, this evidence synthesis was conducted to describe mental health stigma and discrimination in Ethiopia.

\section{Methods \\ The country context}

Ethiopia is a land-locked country located at the horn of Africa. It is the second most populous country in Africa and has a multilingual and multi-ethnic society. The total estimated population was 108,113,150 in 2020 with a growth rate of 2.56\% per annum [30]. The main religious groups are Ethiopian Orthodox Christian 43.5\%, Muslim 33.9\%, Protestant 18.5\%, traditional $2.7 \%$, Catholic $0.7 \%$ and other religions $0.6 \%$ [31].

The health care system in Ethiopia consists of a mixture of public, private and non-governmental health care sectors [14]. There is a three-tiered health care delivery system containing primary health care units (health post, health center and primary hospital), general hospitals, and specialized hospitals [14]. One of the priority areas of the national health policy stated that appropriate support should be given to the curative and rehabilitative components of health, including mental health [32]. The country has also implemented a national mental health strategy 2012/13-2015/16 [33], launched new national mental health strategy 2021-2025 [34], and adopted the WHO Mental Health Gap Action Programme (mhGAP) [35].

\section{Design and approach}

This evidence synthesis was developed based on the cultural adaptation framework of the INDIGO Partnership program to generate the evidence needed to develop culturally and contextually appropriate anti-stigma interventions. We adopted the framework to extract and synthesize records on explanatory models of stigma, perceived and actual stigma levels, the impacts of stigma on help-seeking, and interventions to reduce stigma and discrimination against people with mental illness.

\section{Study selection}

Records in English language published from 2000 onwards about mental health stigma and discrimination in Ethiopia were eligible for this evidence synthesis. In this study, mental illness was operationalized to describe mental health disorders - psychosis/schizophrenia, bipolar disorder anxiety, depression and substance use disorders in particular. The records that contained content related to the domains of the cultural adaptation framework were included. The domains were: explanatory models of stigma (labels for mental illnesses, symptoms and causes of mental illnesses), forms of perceived and experienced stigma, impact of stigma on help-seeking, and interventions to reduce stigma. Stigma was grouped as public, structural, courtesy, and self-stigma based on the situation where prejudice, stereotypes, discrimination or other stigmatizing characteristics occurred. The grouping was based on the definition provided in other studies of stigma $[6,7]$. Accordingly, public stigma is the stigma at general population level in relation to mental health. Courtesy stigma is the stigma experienced by the family/relatives/friends/others that have a relation/proximity to people with mental illness. Structural stigma is the stigma at the level of institutions/organizations/health facilities/social systems due to their policies, laws or regulations regarding mental health. Self-stigma is internalized stigma when stigmatized individuals accept/approve/apply the mentioned stigma characteristics in their life.

\section{Search strategy}

The literature search was conducted through a range of channels to make sure relevant scientific articles as well as grey literature were not missed. The databases used for the search of grey literature (governmental and non-governmental reports, policy documents and issue papers) were Google search and Google scholar. Whereas, for peer reviewed scientific publications the PubMed database was used. The search terms of this evidence synthesis were not necessarily derived from the conceptual framework, rather we used broader approach to capture any literature related to mental health stigma and discrimination. Thus, the relevant concepts for the search were mental health and psychosocial context, stigma and discrimination, mental health system and 
interventions. For each concept the search terms were exhaustively determined. Each concept was combined with the search terms with "AND", whereas the terms in a concept were combined with "OR" in the search engine (Appendix 1).

\section{Record synthesis}

Each record generated from the databases satisfying the eligibility criteria mentioned above was included. Due to the heterogeneity of identified studies, a narrative synthesis was conducted, structured in accordance with the domains of the conceptual framework.

\section{Results}

The initial searches from the electronic databases revealed 13,633 records. Records published before 2000 (1458 records) were excluded. Following the screening, 12,175 records were assessed for eligibility and 12,136 records were excluded (duplicates and irrelevant). The remaining 37 records (2 from grey literature and 35 scientific articles) were synthesized (Fig. 1).

Some of the included records were synthesized more than once for different themes. Thus, 7 addressed explanatory models of stigma (3 records on labels and 4 records on symptoms and causes) and 19 described perceived and experienced forms of stigma (7 records on public stigma, 6 records on structural stigma, 2 records on courtesy stigma and 4 records on self-stigma). The synthesis also included 6 records on the impacts of stigma on help-seeking, and 12 records on interventions to reduce stigma (Table 1). 
Table 1

Synthesized articles

\begin{tabular}{|c|c|c|c|c|c|c|}
\hline $\begin{array}{l}\text { Author, Year and } \\
\text { Journal }\end{array}$ & $\begin{array}{l}\text { Literature } \\
\text { type }\end{array}$ & $\begin{array}{l}\text { Sample size and study } \\
\text { participants }\end{array}$ & Study area & $\begin{array}{l}\text { Measurement } \\
\text { tool/scale }\end{array}$ & $\begin{array}{l}\text { Extracted } \\
\text { themes from } \\
\text { the literature }\end{array}$ & Main finding of the study \\
\hline $\begin{array}{l}\text { Forthal, S., et al. } \\
\text { (2019). (BMC } \\
\text { Psychiatry) }\end{array}$ & $\begin{array}{l}\text { Quantitative } \\
\text { cross- } \\
\text { sectional } \\
\text { study }\end{array}$ & $\begin{array}{l}300 \text { participants with } \\
\text { severe mental illness }\end{array}$ & $\begin{array}{l}\text { Community } \\
\text { based study in } \\
\text { Sodo district, } \\
\text { rural South- } \\
\text { central } \\
\text { Ethiopia }\end{array}$ & $\begin{array}{l}\text { Semi-structured } \\
\text { OPerational } \\
\text { CRITeria for } \\
\text { research } \\
\text { (OPCRIT) } \\
\text { interview, } \\
\text { Discrimination } \\
\text { and Stigma } \\
\text { Scale (DISC12), } \\
\text { Brief Psychiatric } \\
\text { Rating Scale- } \\
\text { Expanded } \\
\text { Version (BPRS- } \\
\text { E), The 36-item } \\
\text { World Health } \\
\text { Organization } \\
\text { Disability } \\
\text { Assessment } \\
\text { Schedule } \\
\text { (WHODAS-2.0) } \\
\text { questionnaire, } \\
\text { Alcohol Use } \\
\text { Disorders } \\
\text { Identification } \\
\text { Test (AUDIT) } \\
\text { self-reported } \\
\text { version, Oslo 3- } \\
\text { item Social } \\
\text { Support Scale } \\
\text { (OSSS-3) }\end{array}$ & $\begin{array}{l}\text { Service users' } \\
\text { perspectives } \\
\text { on public } \\
\text { stigma } \\
\text { against } \\
\text { people with } \\
\text { mental illness }\end{array}$ & $\begin{array}{l}\text { High level of experienced } \\
\text { discrimination and significant } \\
\text { difference was found between urb } \\
\text { and rural people with schizophreni }\end{array}$ \\
\hline $\begin{array}{l}\text { Tesfaw, G., et al. } \\
\text { (2020). } \\
\text { (International } \\
\text { Journal of } \\
\text { Mental Health } \\
\text { Systems) }\end{array}$ & $\begin{array}{l}\text { Quantitative } \\
\text { cross- } \\
\text { sectional } \\
\text { study }\end{array}$ & $\begin{array}{l}423 \text { people with } \\
\text { schizophrenia }\end{array}$ & $\begin{array}{l}\text { Institution } \\
\text { (Amanuel } \\
\text { Mental } \\
\text { Specialized } \\
\text { Hospital) } \\
\text { based study at } \\
\text { Addis Ababa, } \\
\text { Urban Central } \\
\text { Ethiopia }\end{array}$ & $\begin{array}{l}\text { OSSS-3, Positive } \\
\text { and Negative } \\
\text { Syndrome Scale } \\
\text { (PANSS) and } \\
\text { Perceived } \\
\text { Devaluation and } \\
\text { Discrimination } \\
\text { (PDD) Scale. }\end{array}$ & $\begin{array}{l}\text { Service users' } \\
\text { perspectives } \\
\text { on public } \\
\text { stigma } \\
\text { against } \\
\text { people with } \\
\text { mental illness }\end{array}$ & $\begin{array}{l}\text { High perceived stigma among peo } \\
\text { with schizophrenia }\end{array}$ \\
\hline $\begin{array}{l}\text { Girma, E., et al. } \\
\text { (2013). (PLoS } \\
\text { One) }\end{array}$ & $\begin{array}{l}\text { Quantitative } \\
\text { cross- } \\
\text { sectional } \\
\text { study }\end{array}$ & $\begin{array}{l}845 \text { participants from } \\
\text { the community }\end{array}$ & $\begin{array}{l}\text { Community } \\
\text { based study at } \\
\text { Gilgel Gibe } \\
\text { Field Research } \\
\text { Center, Rural } \\
\text { Southwest } \\
\text { Ethiopia }\end{array}$ & $\begin{array}{l}\text { Community } \\
\text { Attitudes } \\
\text { towards the } \\
\text { Mentally III } \\
\text { (CAMI) scale, }\end{array}$ & $\begin{array}{l}\text { Public } \\
\text { perspectives } \\
\text { on public } \\
\text { stigma } \\
\text { against } \\
\text { people with } \\
\text { mental illness }\end{array}$ & $\begin{array}{l}\text { Lower stigma was related with bet } \\
\text { education and perceived explanatc } \\
\text { causes } \\
\text { (supernatural/psychosocial/bioloc } \\
\text { of mental illness }\end{array}$ \\
\hline $\begin{array}{l}\text { Girma, E., et al. } \\
\text { (2013). } \\
\text { (International } \\
\text { Journal of } \\
\text { Mental Health } \\
\text { Systems) }\end{array}$ & $\begin{array}{l}\text { Quantitative } \\
\text { cross- } \\
\text { sectional } \\
\text { study }\end{array}$ & $\begin{array}{l}422 \text { people with severe } \\
\text { mental illness }\end{array}$ & $\begin{array}{l}\text { Institution } \\
\text { (Jimma } \\
\text { University } \\
\text { Specialized } \\
\text { Hospital) } \\
\text { based study at } \\
\text { Jimma town, } \\
\text { Urban } \\
\text { Southwest } \\
\text { Ethiopia }\end{array}$ & $\begin{array}{l}\text { Internalized } \\
\text { Stigma of } \\
\text { Mental Illness } \\
\text { (ISMI) Scale }\end{array}$ & $\begin{array}{l}\text { Service users' } \\
\text { perspectives } \\
\text { on self-stigma } \\
\text { and } \\
\text { interventions } \\
\text { to reduce } \\
\text { stigma } \\
\text { against } \\
\text { people with } \\
\text { mental illness }\end{array}$ & $\begin{array}{l}\text { There was high feeling of inferiorit } \\
\text { but less alignment with common } \\
\text { stereotypes about people with mer } \\
\text { illness. }\end{array}$ \\
\hline $\begin{array}{l}\text { Hadera, E., et al. } \\
\text { (2019). } \\
\text { (Psychiatry } \\
\text { Journal) }\end{array}$ & $\begin{array}{l}\text { Quantitative } \\
\text { cross- } \\
\text { sectional } \\
\text { study }\end{array}$ & $\begin{array}{l}384 \text { outpatient service } \\
\text { users }\end{array}$ & $\begin{array}{l}\text { Institution } \\
\text { (Jimma } \\
\text { University } \\
\text { Specialized } \\
\text { Hospital) } \\
\text { based study at } \\
\text { Jimma town, } \\
\text { Urban } \\
\text { Southwest } \\
\text { Ethiopia) } \\
\text { based study }\end{array}$ & $\begin{array}{l}\text { Perceived } \\
\text { Devaluation and } \\
\text { Discrimination } \\
\text { (PDD) scale }\end{array}$ & $\begin{array}{l}\text { Service users' } \\
\text { perspectives } \\
\text { on public } \\
\text { stigma } \\
\text { against } \\
\text { people with } \\
\text { mental illness }\end{array}$ & $\begin{array}{l}\text { Perceived prevailing stigma amon } \\
\text { outpatient mental health care serv } \\
\text { users }\end{array}$ \\
\hline $\begin{array}{l}\text { Reta, Y., et al. } \\
\text { (2016). (PLoS } \\
\text { One) }\end{array}$ & $\begin{array}{l}\text { Quantitative } \\
\text { cross- } \\
\text { sectional } \\
\text { study }\end{array}$ & $\begin{array}{l}820 \text { participants from } \\
\text { the community }\end{array}$ & $\begin{array}{l}\text { Community } \\
\text { based study at } \\
\text { Jimma town, } \\
\text { Urban } \\
\text { Southwest } \\
\text { Ethiopia }\end{array}$ & $\begin{array}{l}\text { Community } \\
\text { Attitudes toward } \\
\text { the Mentally III } \\
\text { (CAMI) scale }\end{array}$ & $\begin{array}{l}\text { Public } \\
\text { perspectives } \\
\text { on public } \\
\text { stigma } \\
\text { against } \\
\text { people with } \\
\text { mental illness }\end{array}$ & $\begin{array}{l}\text { Prevailing negative attitude agains } \\
\text { people with mental illness }\end{array}$ \\
\hline
\end{tabular}




\begin{tabular}{|c|c|c|c|c|c|c|}
\hline $\begin{array}{l}\text { Author, Year and } \\
\text { Journal }\end{array}$ & $\begin{array}{l}\text { Literature } \\
\text { type }\end{array}$ & $\begin{array}{l}\text { Sample size and study } \\
\text { participants }\end{array}$ & Study area & $\begin{array}{l}\text { Measurement } \\
\text { tool/scale }\end{array}$ & $\begin{array}{l}\text { Extracted } \\
\text { themes from } \\
\text { the literature }\end{array}$ & Main finding of the study \\
\hline $\begin{array}{l}\text { Hailemariam, } \\
\text { M., et al. (2017). } \\
\text { (Int J Equity } \\
\text { Health) }\end{array}$ & $\begin{array}{l}\text { Qualitative } \\
\text { study }\end{array}$ & $\begin{array}{l}50 \text { In-depth interviews } \\
\text { with service users, care } \\
\text { givers and service } \\
\text { providers in the facility } \\
\text { and } 2 \text { FGDs ( } 10 \text { per } \\
\text { group) with community } \\
\text { service providers }\end{array}$ & $\begin{array}{l}\text { Community } \\
\text { based study in } \\
\text { Sodo district, } \\
\text { rural South- } \\
\text { central } \\
\text { Ethiopia }\end{array}$ & $\begin{array}{l}\text { Interview guide } \\
\text { was developed } \\
\text { for countries } \\
\text { implementing } \\
\text { PRIME }\end{array}$ & $\begin{array}{l}\text { Service users', } \\
\text { caregivers' } \\
\text { and service } \\
\text { providers' } \\
\text { perspectives } \\
\text { on public } \\
\text { stigma } \\
\text { against } \\
\text { people with } \\
\text { mental illness }\end{array}$ & $\begin{array}{l}\text { Financial problems and in accessi } \\
\text { of mental health facility and the } \\
\text { medication were the main barriers } \\
\text { the engagement to care' }\end{array}$ \\
\hline $\begin{array}{l}\text { Abayneh, } \\
\text { Lempp et al. } \\
\text { (2017). (BMC } \\
\text { Psychiatry) }\end{array}$ & $\begin{array}{l}\text { Qualitative } \\
\text { study }\end{array}$ & $\begin{array}{l}39 \text { interviews with } \\
\text { service users, care } \\
\text { givers, managers and } \\
\text { policy } \\
\text { makers/planners/service } \\
\text { developers at national } \\
\text { and regional level }\end{array}$ & $\begin{array}{l}\text { Community } \\
\text { based study at } \\
\text { Butajira (rural } \\
\text { central part of } \\
\text { Ethiopia) and } \\
\text { with national } \\
\text { representatives }\end{array}$ & $\begin{array}{l}\text { Interview guide } \\
\text { was developed } \\
\text { for Emerging } \\
\text { mental health } \\
\text { systems in low- } \\
\text { and middle- } \\
\text { income } \\
\text { countries } \\
\text { (Emerald) cross- } \\
\text { country study }\end{array}$ & $\begin{array}{l}\text { Service users', } \\
\text { caregivers' } \\
\text { and service } \\
\text { providers' } \\
\text { perspectives } \\
\text { on public } \\
\text { stigma, } \\
\text { structural } \\
\text { stigma and } \\
\text { intervention } \\
\text { on stigma }\end{array}$ & $\begin{array}{l}\text { Despite the increasing access of } \\
\text { mental health care, there were barr } \\
\text { in relation to stigma and awarenes }\end{array}$ \\
\hline $\begin{array}{l}\text { Hanlon, C., et al. } \\
\text { (2017). } \\
\text { (International } \\
\text { Journal of } \\
\text { Mental Health } \\
\text { Systems) }\end{array}$ & $\begin{array}{l}\text { Qualitative } \\
\text { study }\end{array}$ & $\begin{array}{l}17 \text { in-depth interviews } \\
\text { with national and } \\
\text { regional policy makers, } \\
\text { service developers and } \\
\text { district level health office } \\
\text { managers. }\end{array}$ & $\begin{array}{l}\text { Community } \\
\text { based study in } \\
\text { Sodo district, } \\
\text { rural South- } \\
\text { central } \\
\text { Ethiopia }\end{array}$ & $\begin{array}{l}\text { Interview guide } \\
\text { was derived } \\
\text { from the } \\
\text { adapted Health } \\
\text { System } \\
\text { Governance } \\
\text { (HSG) } \\
\text { evaluation } \\
\text { framework }\end{array}$ & $\begin{array}{l}\text { Policy } \\
\text { makers' and } \\
\text { service } \\
\text { developers' } \\
\text { perspectives } \\
\text { on structural } \\
\text { stigma. }\end{array}$ & $\begin{array}{l}\text { There are improved government } \\
\text { support and commitment to scale- } \\
\text { mental health care. However, the } \\
\text { leadership and coordination amon } \\
\text { different administrative levels neer } \\
\text { be strengthened in addition to } \\
\text { addressing awareness problems }\end{array}$ \\
\hline $\begin{array}{l}\text { Hailemariam, } \\
\text { M., et al. (2019). } \\
\text { (International } \\
\text { Journal of } \\
\text { Mental Health } \\
\text { Systems) }\end{array}$ & $\begin{array}{l}\text { Quantitative } \\
\text { cross- } \\
\text { sectional } \\
\text { study }\end{array}$ & $\begin{array}{l}369 \text { participants with } \\
\text { probable cases of severe } \\
\text { mental illness }\end{array}$ & $\begin{array}{l}\text { Community } \\
\text { based study in } \\
\text { Sodo district, } \\
\text { rural South- } \\
\text { central } \\
\text { Ethiopia }\end{array}$ & $\begin{array}{l}\text { Barriers to } \\
\text { Access to Care } \\
\text { Evaluation } \\
\text { (BACE-3), Short } \\
\text { Explanatory } \\
\text { Model Interview } \\
\text { (SEMI), WHO } 12 \\
\text { item Disability } \\
\text { Assessment } \\
\text { Schedule } \\
\text { (WHODAS 2.0), } \\
\text { Oslo-3 scale, } \\
\text { Alcohol Use } \\
\text { Disorders } \\
\text { Identification } \\
\text { Test (AUDIT), } \\
\text { Discrimination } \\
\text { and Stigma } \\
\text { Scale (DISC-12), } \\
\text { Brief Physical } \\
\text { Impairment } \\
\text { Rating Checklist } \\
\text { (BPIRC), } \\
\text { Washington } \\
\text { Group's } \\
\text { disability } \\
\text { measure and } \\
\text { Work, Family } \\
\text { and Well-being } \\
\text { (WFW) scales. }\end{array}$ & $\begin{array}{l}\text { Service users' } \\
\text { perspectives } \\
\text { on structural } \\
\text { stigma, } \\
\text { interventions } \\
\text { to reduce } \\
\text { stigma and } \\
\text { impact of } \\
\text { stigma on } \\
\text { help-seeking } \\
\text { of people with } \\
\text { severe mental } \\
\text { illness. }\end{array}$ & $\begin{array}{l}\text { Integrating mental health care into } \\
\text { primary care highly improves the Iı } \\
\text { of equitable contact coverage. }\end{array}$ \\
\hline $\begin{array}{l}\text { Abayneh, S., et } \\
\text { al. (2020). } \\
\text { (International } \\
\text { Journal of } \\
\text { Mental Health } \\
\text { Systems) }\end{array}$ & $\begin{array}{l}\text { Qualitative } \\
\text { study, Theory } \\
\text { of Change } \\
\text { (ToC) } \\
\text { development }\end{array}$ & $\begin{array}{l}31 \text { participants (4 } \\
\text { psychiatrists, } 3 \\
\text { researchers, } 24 \\
\text { stakeholders involved in } \\
\text { service planning) }\end{array}$ & $\begin{array}{l}\text { Community } \\
\text { and institution- } \\
\text { based study at } \\
\text { sodo district } \\
\text { (rural south- } \\
\text { central } \\
\text { Ethiopia) and } \\
\text { Addis Ababa } \\
\text { (urban central } \\
\text { Ethiopia) } \\
\text { respectively }\end{array}$ & $\begin{array}{l}\text { ToC Maps, } \\
\text { workshop and } \\
\text { meeting minutes }\end{array}$ & $\begin{array}{l}\text { Service } \\
\text { providers' and } \\
\text { service } \\
\text { developers' } \\
\text { and } \\
\text { administers' } \\
\text { perspectives } \\
\text { on structural } \\
\text { stigma and } \\
\text { interventions } \\
\text { to reduce } \\
\text { stigma. }\end{array}$ & $\begin{array}{l}\text { In order to mobilize and empower } \\
\text { service users and caregivers for } \mathrm{m} \\
\text { health system improvement, the } \\
\text { service user and caregiver progran } \\
\text { level was } \\
\text { the main intervention component } \\
\text { identified in the ToC. }\end{array}$ \\
\hline
\end{tabular}




\begin{tabular}{|c|c|c|c|c|c|c|}
\hline $\begin{array}{l}\text { Author, Year and } \\
\text { Journal }\end{array}$ & $\begin{array}{l}\text { Literature } \\
\text { type }\end{array}$ & $\begin{array}{l}\text { Sample size and study } \\
\text { participants }\end{array}$ & Study area & $\begin{array}{l}\text { Measurement } \\
\text { tool/scale }\end{array}$ & $\begin{array}{l}\text { Extracted } \\
\text { themes from } \\
\text { the literature }\end{array}$ & Main finding of the study \\
\hline $\begin{array}{l}\text { Girma, E., et al. } \\
(2014) \text {. (Journal } \\
\text { of } \\
\text { multidisciplinary } \\
\text { healthcare) }\end{array}$ & $\begin{array}{l}\text { Quantitative } \\
\text { cross- } \\
\text { sectional } \\
\text { study }\end{array}$ & $\begin{array}{l}422 \text { participants who } \\
\text { were caregivers }\end{array}$ & $\begin{array}{l}\text { Institution } \\
\text { (Jimma } \\
\text { University } \\
\text { Specialized } \\
\text { Hospital) } \\
\text { based study at } \\
\text { Jimma town, } \\
\text { Urban } \\
\text { Southwest } \\
\text { Ethiopia) } \\
\text { based study }\end{array}$ & $\begin{array}{l}\text { Questionnaire } \\
\text { adopted from } \\
\text { WHO Family } \\
\text { Interview } \\
\text { Schedule stigma } \\
\text { items and other } \\
\text { literatures }\end{array}$ & $\begin{array}{l}\text { Care givers' } \\
\text { perspectives } \\
\text { on self-stigma } \\
\text { due to family } \\
\text { members' } \\
\text { mental illness }\end{array}$ & $\begin{array}{l}\text { Even though the care givers' self- } \\
\text { stigma was found to be low, it mig } \\
\text { have an impact on help seeking } \\
\text { behavior of people mental illness }\end{array}$ \\
\hline $\begin{array}{l}\text { Girma, E., et al. } \\
(2014) \text {. (BMC } \\
\text { international } \\
\text { health and } \\
\text { human rights) }\end{array}$ & $\begin{array}{l}\text { Quantitative } \\
\text { cross- } \\
\text { sectional } \\
\text { study }\end{array}$ & $\begin{array}{l}845 \text { participants from } \\
\text { the community }\end{array}$ & $\begin{array}{l}\text { Community } \\
\text { based study at } \\
\text { Gilgel Gibe } \\
\text { Field Research } \\
\text { Center, Rural } \\
\text { Southwest } \\
\text { Ethiopia }\end{array}$ & $\begin{array}{l}\text { Questionnaire } \\
\text { adopted from } \\
\text { Devaluation of } \\
\text { Consumer } \\
\text { Families Scale } \\
\text { and other } \\
\text { literatures }\end{array}$ & $\begin{array}{l}\text { Care givers' } \\
\text { perspectives } \\
\text { on public } \\
\text { stigma due to } \\
\text { family } \\
\text { members' } \\
\text { mental illness }\end{array}$ & $\begin{array}{l}\text { There was moderate level of perce } \\
\text { public stigma against family mem } \\
\text { of PWMI and it was negatively } \\
\text { correlated with perceived sympton } \\
\text { and explanatory causes } \\
\text { (supernatural/psychosocial/biolos } \\
\text { of mental illness. }\end{array}$ \\
\hline $\begin{array}{l}\text { Assefa, D., et al. } \\
\text { (2012). (BMC } \\
\text { Psychiatry) }\end{array}$ & $\begin{array}{l}\text { Quantitative } \\
\text { cross- } \\
\text { sectional } \\
\text { study }\end{array}$ & $\begin{array}{l}212 \text { individuals with } \\
\text { schizophrenia }\end{array}$ & $\begin{array}{l}\text { Institution } \\
\text { (Amanuel } \\
\text { Mental } \\
\text { Specialized } \\
\text { Hospital) } \\
\text { based study at } \\
\text { Addis Ababa, } \\
\text { Urban Central } \\
\text { Ethiopia }\end{array}$ & $\begin{array}{l}\text { Internalized } \\
\text { Stigma of } \\
\text { Mental Illness } \\
\text { (ISMI) Scale and } \\
\text { Diagnostic and } \\
\text { Statistical } \\
\text { Manual of } \\
\text { Mental } \\
\text { Disorders, } \\
\text { Fourth edition } \\
\text { (DSM-IV) }\end{array}$ & $\begin{array}{l}\text { Service users' } \\
\text { perspectives } \\
\text { towards self- } \\
\text { stigma } \\
\text { among people } \\
\text { with } \\
\text { schizophrenia }\end{array}$ & $\begin{array}{l}\text { Internalized stigma was indicated } \\
\text { the prevailing problem among peo } \\
\text { with schizophrenia as nearly half ( } \\
\text { them had the problem }\end{array}$ \\
\hline $\begin{array}{l}\text { Bifftu, B. B., et } \\
\text { al. (2014). (BMC } \\
\text { Psychiatry) }\end{array}$ & $\begin{array}{l}\text { Quantitative } \\
\text { cross- } \\
\text { sectional } \\
\text { study }\end{array}$ & $\begin{array}{l}411 \text { individuals with } \\
\text { schizophrenia }\end{array}$ & $\begin{array}{l}\text { Institution } \\
\text { (Amanuel } \\
\text { Mental } \\
\text { Specialized } \\
\text { Hospital) } \\
\text { based study at } \\
\text { Addis Ababa, } \\
\text { Urban Central } \\
\text { Ethiopia }\end{array}$ & $\begin{array}{l}\text { Internalized } \\
\text { Stigma of } \\
\text { Mental Illness } \\
\text { (ISMI) Scale }\end{array}$ & $\begin{array}{l}\text { Service users' } \\
\text { perspectives } \\
\text { towards self- } \\
\text { stigma } \\
\text { among people } \\
\text { with } \\
\text { schizophrenia }\end{array}$ & $\begin{array}{l}\text { Low stigma resistance was reportt } \\
\text { nearly half of the participants with } \\
\text { schizophrenia }\end{array}$ \\
\hline $\begin{array}{l}\text { Zewdu, S., et al. } \\
\text { (2019). } \\
\text { (Substance } \\
\text { Abuse } \\
\text { Treatment, } \\
\text { Prevention, and } \\
\text { Policy) }\end{array}$ & $\begin{array}{l}\text { Quantitative } \\
\text { cross- } \\
\text { sectional } \\
\text { study }\end{array}$ & $\begin{array}{l}1500 \text { adults from the } \\
\text { community }\end{array}$ & $\begin{array}{l}\text { Community } \\
\text { based study in } \\
\text { Sodo district, } \\
\text { rural South- } \\
\text { central } \\
\text { Ethiopia }\end{array}$ & $\begin{array}{l}\text { Alcohol Use } \\
\text { Disorder } \\
\text { Identification } \\
\text { Tool (AUDIT), } \\
\text { Patient Health } \\
\text { Questionnaire } \\
\text { (PHQ-9) for } \\
\text { depression, } \\
\text { WHO Composite } \\
\text { International } \\
\text { Diagnostic } \\
\text { Interview (CIDI), } \\
\text { WHO Disability } \\
\text { Assessment } \\
\text { Schedule } \\
\text { (WHODAS) } \\
\text { version 2.0, with } \\
\text { 12 items, List of } \\
\text { Threatening } \\
\text { Experiences } \\
\text { (LTE) } \\
\text { questionnaire, 3- } \\
\text { item Oslo Social } \\
\text { Support (OSS) } \\
\text { questionnaire } \\
\text { and Internalized } \\
\text { Stigma of } \\
\text { Mental IIIness } \\
\text { Inventory (ISMI) }\end{array}$ & $\begin{array}{l}\text { Service users' } \\
\text { perspectives } \\
\text { towards self- } \\
\text { stigma } \\
\text { among people } \\
\text { with alcohol } \\
\text { use disorder }\end{array}$ & $\begin{array}{l}\text { The twelve-month prevalence of } \\
\text { alcohol use disorder was } 13.9 \% \text { ar } \\
\text { most of whom didn't seek medical } \\
\text { and had high internalized stigma. }\end{array}$ \\
\hline $\begin{array}{l}\text { Asher, L., et al. } \\
\text { (2017). } \\
\text { (Globalization } \\
\text { and Health) }\end{array}$ & $\begin{array}{l}\text { Qualitative } \\
\text { study (RISE } \\
\text { study) }\end{array}$ & $\begin{array}{l}35 \text { participants (5 in- } \\
\text { depth interviews and } 5 \\
\text { focus group } \\
\text { discussions) composed } \\
\text { of people with } \\
\text { schizophrenia, their } \\
\text { caregivers, community } \\
\text { leaders and primary and } \\
\text { community service } \\
\text { providers }\end{array}$ & $\begin{array}{l}\text { Community } \\
\text { based study in } \\
\text { Sodo district, } \\
\text { rural South- } \\
\text { central } \\
\text { Ethiopia }\end{array}$ & $\begin{array}{l}\text { Used topic guide } \\
\text { as part of } \\
\text { Rehabilitation } \\
\text { Intervention for } \\
\text { people with } \\
\text { Schizophrenia in } \\
\text { Ethiopia (RISE) } \\
\text { project. }\end{array}$ & $\begin{array}{l}\text { Care givers' } \\
\text { perspectives } \\
\text { on the impact } \\
\text { of stigma on } \\
\text { help-seeking } \\
\text { behavior of } \\
\text { people with } \\
\text { mental illness }\end{array}$ & $\begin{array}{l}\text { Physical restraint of people with } \\
\text { schizophrenia was common pract } \\
\text { which could restrict them from } \\
\text { attending the needed medical care }\end{array}$ \\
\hline
\end{tabular}




\begin{tabular}{|c|c|c|c|c|c|c|}
\hline $\begin{array}{l}\text { Author, Year and } \\
\text { Journal }\end{array}$ & $\begin{array}{l}\text { Literature } \\
\text { type }\end{array}$ & $\begin{array}{l}\text { Sample size and study } \\
\text { participants }\end{array}$ & Study area & $\begin{array}{l}\text { Measurement } \\
\text { tool/scale }\end{array}$ & $\begin{array}{l}\text { Extracted } \\
\text { themes from } \\
\text { the literature }\end{array}$ & Main finding of the study \\
\hline $\begin{array}{l}\text { Fekadu, A., et al. } \\
\text { (2019). (BMC } \\
\text { Psychiatry) }\end{array}$ & $\begin{array}{l}\text { Quantitative } \\
\text { cross- } \\
\text { sectional } \\
\text { study }\end{array}$ & $\begin{array}{l}300 \text { participants with } \\
\text { psychosis }\end{array}$ & $\begin{array}{l}\text { Community } \\
\text { based study in } \\
\text { Butajira, rural } \\
\text { central part of } \\
\text { Ethiopia }\end{array}$ & $\begin{array}{l}\text { The Butajira } \\
\text { Treatment Gap } \\
\text { Questionnaire } \\
\text { (TGQ), Brief } \\
\text { Psychiatric } \\
\text { Rating Scale- } \\
\text { Expanded } \\
\text { version (BPRS- } \\
\text { E), WHO } \\
\text { Disability } \\
\text { Assessment } \\
\text { Schedule } \\
\text { (WHODAS 2.0), } \\
\text { Oslo } 3 \text { Social } \\
\text { Support Scale } \\
\text { (OSS), } \\
\text { Operational } \\
\text { Criteria for } \\
\text { Research } \\
\text { (OPCRIT) }\end{array}$ & $\begin{array}{l}\text { Service users' } \\
\text { perspectives } \\
\text { towards the } \\
\text { impact of } \\
\text { stigma on } \\
\text { help-seeking } \\
\text { behavior of } \\
\text { people with } \\
\text { mental illness }\end{array}$ & $\begin{array}{l}\text { Nearly six out of ten participants h } \\
\text { current access gap to biomedical ( } \\
\text { which could affect their work func } \\
\text { and might experience discriminati }\end{array}$ \\
\hline $\begin{array}{l}\text { Souraya, S., et } \\
\text { al. (2018). } \\
\text { (Globalization } \\
\text { and Health) }\end{array}$ & $\begin{array}{l}\text { Qualitative } \\
\text { study }\end{array}$ & $\begin{array}{l}18 \text { in depth interviews } \\
\text { (people with } \\
\text { schizophrenia, } \\
\text { caregivers, service } \\
\text { providers and officials) } \\
\text { and } 2 \text { FGDs with } \\
\text { community- based } \\
\text { rehabilitation workers }\end{array}$ & $\begin{array}{l}\text { Community } \\
\text { based study in } \\
\text { Sodo district, } \\
\text { rural South- } \\
\text { central } \\
\text { Ethiopia }\end{array}$ & $\begin{array}{l}\text { Used topic guide } \\
\text { as part of } \\
\text { Rehabilitation } \\
\text { Intervention for } \\
\text { people with } \\
\text { Schizophrenia in } \\
\text { Ethiopia (RISE) } \\
\text { project. }\end{array}$ & $\begin{array}{l}\text { Service users' } \\
\text { and } \\
\text { caregivers' } \\
\text { perspectives } \\
\text { towards the } \\
\text { impact of } \\
\text { stigma on } \\
\text { help-seeking } \\
\text { behavior of } \\
\text { people with } \\
\text { mental illness }\end{array}$ & $\begin{array}{l}\text { It is not common practice to involv } \\
\text { service users and care givers in } \\
\text { decision making towards the treat } \\
\text { options }\end{array}$ \\
\hline $\begin{array}{l}\text { Girma, E. and M. } \\
\text { Tesfaye (2011). } \\
\text { (BMC } \\
\text { Psychiatry) }\end{array}$ & $\begin{array}{l}\text { Quantitative } \\
\text { cross- } \\
\text { sectional } \\
\text { study }\end{array}$ & $\begin{array}{l}384 \text { participants with } \\
\text { psychosis }\end{array}$ & $\begin{array}{l}\text { Institution } \\
\text { (Jimma } \\
\text { University } \\
\text { Specialized } \\
\text { Hospital) } \\
\text { based study at } \\
\text { Jimma town, } \\
\text { Urban } \\
\text { Southwest } \\
\text { Ethiopia }\end{array}$ & $\begin{array}{l}\text { Questionnaire } \\
\text { was developed } \\
\text { based on WHO } \\
\text { Encounter Form } \\
\text { for Pathways to } \\
\text { Care and Good's } \\
\text { Pathway Model }\end{array}$ & $\begin{array}{l}\text { Service users' } \\
\text { perspectives } \\
\text { towards the } \\
\text { impact of } \\
\text { stigma on } \\
\text { help-seeking } \\
\text { behavior of } \\
\text { people with } \\
\text { mental illness }\end{array}$ & $\begin{array}{l}\text { There was significant delay in } \\
\text { treatment seeking behavior amons } \\
\text { people with psychosis }\end{array}$ \\
\hline $\begin{array}{l}\text { Tsigebrhan, R., } \\
\text { et al. (2014). } \\
\text { (Schizophrenia } \\
\text { Research) }\end{array}$ & $\begin{array}{l}\text { Quantitative } \\
\text { comparative } \\
\text { cross- } \\
\text { sectional } \\
\text { study }\end{array}$ & $\begin{array}{l}201 \text { participants with } \\
\text { severe mental illness }\end{array}$ & $\begin{array}{l}\text { Community } \\
\text { based study in } \\
\text { Butajira, rural } \\
\text { central part of } \\
\text { Ethiopia }\end{array}$ & $\begin{array}{l}\text { Questionnaire } \\
\text { was prepared } \\
\text { based on the } \\
\text { MacArthur } \\
\text { Violence } \\
\text { interview, } \\
\text { Modified version } \\
\text { of the Historical, } \\
\text { Clinical and Risk } \\
\text { management } \\
\text { (HCR-20) scale } \\
\text { and other } \\
\text { literatures }\end{array}$ & $\begin{array}{l}\text { Service users' } \\
\text { perspectives } \\
\text { towards the } \\
\text { impact of } \\
\text { stigma on } \\
\text { help-seeking } \\
\text { behavior of } \\
\text { people with } \\
\text { mental illness }\end{array}$ & $\begin{array}{l}\text { There significantly higher level of } \\
\text { violence and violence victimizatior } \\
\text { among people with severe mental } \\
\text { illness which alarms the need } \\
\text { improvement in mental health cart } \\
\text { access. }\end{array}$ \\
\hline $\begin{array}{l}\text { Tirfessa, K., et } \\
\text { al. (2020). } \\
\text { (Tropical } \\
\text { medicine \& } \\
\text { international } \\
\text { health) }\end{array}$ & $\begin{array}{l}\text { Quantitative } \\
\text { controlled } \\
\text { before-after } \\
\text { study }\end{array}$ & $\begin{array}{l}239 \text { participants with } \\
\text { severe mental illness } \\
\text { and } 273 \text { participants as } \\
\text { control households }\end{array}$ & $\begin{array}{l}\text { Community } \\
\text { based study in } \\
\text { Sodo district, } \\
\text { rural South- } \\
\text { central } \\
\text { Ethiopia }\end{array}$ & $\begin{array}{l}\text { Household Food } \\
\text { Insecurity } \\
\text { Access Scale } \\
\text { (HFIAS), } \\
\text { Discrimination } \\
\text { and Stigma } \\
\text { Scale-12 (DISC- } \\
\text { 12), WHO } \\
\text { Disability } \\
\text { Assessment } \\
\text { Schedule } \\
\text { (WHODAS) } 2.0 \\
\text { 12-item version, } \\
\text { Longitudinal } \\
\text { Interval Follow- } \\
\text { up Evaluation- } \\
\text { Range of } \\
\text { Impaired } \\
\text { Functioning Tool } \\
\text { (LIFE-RIFT) and } \\
\text { 24-item Brief } \\
\text { Psychiatric } \\
\text { Rating Scale- } \\
\text { Expanded } \\
\text { version (BPRS- } \\
\text { E). }\end{array}$ & $\begin{array}{l}\text { Service users' } \\
\text { perspectives } \\
\text { towards } \\
\text { interventions } \\
\text { to reduce } \\
\text { stigma } \\
\text { against } \\
\text { people with } \\
\text { mental illness }\end{array}$ & $\begin{array}{l}\text { The improvement in access to me } \\
\text { health care led to improvement in } \\
\text { households' food security of more } \\
\text { half of the households with peopl } \\
\text { with severe mental illness. }\end{array}$ \\
\hline
\end{tabular}




\begin{tabular}{|c|c|c|c|c|c|c|}
\hline $\begin{array}{l}\text { Author, Year and } \\
\text { Journal }\end{array}$ & $\begin{array}{l}\text { Literature } \\
\text { type }\end{array}$ & $\begin{array}{l}\text { Sample size and study } \\
\text { participants }\end{array}$ & Study area & $\begin{array}{l}\text { Measurement } \\
\text { tool/scale }\end{array}$ & $\begin{array}{l}\text { Extracted } \\
\text { themes from } \\
\text { the literature }\end{array}$ & Main finding of the study \\
\hline $\begin{array}{l}\text { Habtamu, K., et } \\
\text { al. (2018). } \\
\text { (Social } \\
\text { psychiatry and } \\
\text { psychiatric } \\
\text { epidemiology) }\end{array}$ & $\begin{array}{l}\text { Quantitative } \\
\text { cross- } \\
\text { sectional } \\
\text { study }\end{array}$ & $\begin{array}{l}324 \text { participants with } \\
\text { severe mental illness }\end{array}$ & $\begin{array}{l}\text { Community } \\
\text { based study at } \\
\text { Butajira, rural } \\
\text { central part of } \\
\text { Ethiopia }\end{array}$ & $\begin{array}{l}\text { WHO Disability } \\
\text { Assessment } \\
\text { Schedule } \\
\text { (WHODAS-2.0), } \\
\text { Butajira } \\
\text { Functioning } \\
\text { Scale (BFS), } \\
\text { Brief Psychiatric } \\
\text { Rating Scale } \\
\text { (BPRS-E), } \\
\text { Composite } \\
\text { International } \\
\text { Diagnostic } \\
\text { Interview (CIDI) } \\
\text { substance } \\
\text { abuse module, } \\
\text { Mini } \\
\text { International } \\
\text { Neuropsychiatric } \\
\text { Interview (MINI) } \\
\text { Suicidality } \\
\text { Scale, } \\
\text { Antipsychotic } \\
\text { Side effects } \\
\text { Checklist (ASC), } \\
\text { Life Chart } \\
\text { Schedule (LCS), } \\
\text { Internalized } \\
\text { Stigma of } \\
\text { Mental Illness } \\
\text { (ISMI) scale }\end{array}$ & $\begin{array}{l}\text { Service users' } \\
\text { perspectives } \\
\text { towards } \\
\text { interventions } \\
\text { to reduce } \\
\text { stigma } \\
\text { against } \\
\text { people with } \\
\text { mental illness }\end{array}$ & $\begin{array}{l}\text { Functional impairment was detern } \\
\text { by symptom severity, poverty, } \\
\text { medication side effects, and } \\
\text { internalized stigma among people } \\
\text { severe mental illness }\end{array}$ \\
\hline $\begin{array}{l}\text { Asher, L., et al. } \\
(2015) \text { (PloS } \\
\text { One) }\end{array}$ & $\begin{array}{l}\text { Qualitative } \\
\text { study }\end{array}$ & $\begin{array}{l}\text { Two consecutive } \\
\text { workshops with } 8 \\
\text { mental health experts } \\
\text { and } 12 \text { community } \\
\text { leaders respectively, } 16 \\
\text { in-depth interviews and } 5 \\
\text { FGDs with service users } \\
\text { (people with } \\
\text { schizophrenia), } \\
\text { caregivers and service } \\
\text { providers }\end{array}$ & $\begin{array}{l}\text { Intervention } \\
\text { development } \\
\text { work in Sodo } \\
\text { district, rural } \\
\text { South-central } \\
\text { Ethiopia }\end{array}$ & $\begin{array}{l}\text { workshop notes, } \\
\text { site visits and in- } \\
\text { depth } \\
\text { consultation, } \\
\text { data extraction } \\
\text { and interview } \\
\text { guide }\end{array}$ & $\begin{array}{l}\text { Service users', } \\
\text { care givers' } \\
\text { and providers' } \\
\text { perspectives } \\
\text { towards } \\
\text { interventions } \\
\text { to reduce } \\
\text { stigma } \\
\text { against } \\
\text { people with } \\
\text { mental illness }\end{array}$ & $\begin{array}{l}\text { People with schizophrenia have } \\
\text { challenges in maintaining work } \\
\text { function, social and family role an } \\
\text { experienced stigma which are } \\
\text { perceived to be addressed with } \\
\text { culturally appropriate, acceptable } \\
\text { feasible community-based } \\
\text { rehabilitation intervention. }\end{array}$ \\
\hline $\begin{array}{l}\text { Asher, L., et al. } \\
\text { (2018). (BMC } \\
\text { Psychiatry) }\end{array}$ & $\begin{array}{l}\text { Follow up } \\
\text { Mixed } \\
\text { method } \\
\text { (Qualitative } \\
\text { and } \\
\text { Quantitative) } \\
\text { pilot study }\end{array}$ & $\begin{array}{l}31 \text { individuals (people } \\
\text { with schizophrenia, } \\
\text { caregivers, community } \\
\text { members and service } \\
\text { providers) }\end{array}$ & $\begin{array}{l}\text { Community } \\
\text { based pilot } \\
\text { study at Sodo } \\
\text { district, rural } \\
\text { South-central } \\
\text { Ethiopia }\end{array}$ & $\begin{array}{l}\text { Used topic } \\
\text { guide, data } \\
\text { extraction form, } \\
\text { and } \\
\text { measurement } \\
\text { scales; } \\
\text { Discrimination } \\
\text { and Stigma } \\
\text { Scale-12 (DISC- } \\
\text { 12) subscale 1, } \\
\text { Alcohol Use } \\
\text { Disorders } \\
\text { Identification } \\
\text { Test (AUDIT), } \\
\text { Patient Health } \\
\text { Questionnaire-9 } \\
\text { (PHQ-9), } \\
\text { Involvement } \\
\text { Evaluation } \\
\text { Questionnaire } \\
\text { (IEQ), WHO } \\
\text { Disability } \\
\text { Assessment } \\
\text { Schedule } \\
\text { (WHODAS) 2.0 } \\
\text { and Clinical } \\
\text { Global } \\
\text { Impression (CGI) }\end{array}$ & $\begin{array}{l}\text { Service users', } \\
\text { care givers' } \\
\text { and providers' } \\
\text { perspectives } \\
\text { towards } \\
\text { interventions } \\
\text { to reduce } \\
\text { stigma } \\
\text { against } \\
\text { people with } \\
\text { mental illness }\end{array}$ & $\begin{array}{l}\text { Community-based rehabilitation } \\
\text { intervention was proven to be } \\
\text { acceptable and feasible approach } \\
\text { which should be used with the exis } \\
\text { facility-based care for people with } \\
\text { schizophrenia }\end{array}$ \\
\hline
\end{tabular}




\begin{tabular}{|c|c|c|c|c|c|c|}
\hline $\begin{array}{l}\text { Author, Year and } \\
\text { Journal }\end{array}$ & $\begin{array}{l}\text { Literature } \\
\text { type }\end{array}$ & $\begin{array}{l}\text { Sample size and study } \\
\text { participants }\end{array}$ & Study area & $\begin{array}{l}\text { Measurement } \\
\text { tool/scale }\end{array}$ & $\begin{array}{l}\text { Extracted } \\
\text { themes from } \\
\text { the literature }\end{array}$ & Main finding of the study \\
\hline $\begin{array}{l}\text { Hanlon, C., et al. } \\
\text { (2019). } \\
\text { (Epidemiology } \\
\text { and psychiatric } \\
\text { sciences) }\end{array}$ & $\begin{array}{l}\text { Quantitative } \\
\text { interventional } \\
\text { cohort study } \\
\text { (PRIME } \\
\text { study) }\end{array}$ & $\begin{array}{l}245 \text { participants with } \\
\text { severe mental illness }\end{array}$ & $\begin{array}{l}\text { Institution } \\
\text { based study at } \\
\text { Sodo district, } \\
\text { rural South- } \\
\text { central } \\
\text { Ethiopia }\end{array}$ & $\begin{array}{l}\text { Brief Psychiatric } \\
\text { Rating Scale, } \\
\text { expanded } \\
\text { version (BPRS- } \\
\text { E), WHO } \\
\text { Disability } \\
\text { Assessment } \\
\text { Schedule } \\
\text { (WHODAS), } \\
\text { 'unfair } \\
\text { treatment' } \\
\text { subscale of the } \\
\text { Discrimination } \\
\text { and Stigma } \\
\text { Scale-12 (DISC- } \\
\text { 12), Alcohol Use } \\
\text { Disorder } \\
\text { Identification } \\
\text { Test (AUDIT), } \\
\text { locally validated } \\
\text { version of the } \\
\text { Patient Health } \\
\text { Questionnaire } \\
\text { (PHQ-9) and } \\
\text { three-item Oslo } \\
\text { Social Support } \\
\text { Scale (OSS-3) }\end{array}$ & $\begin{array}{l}\text { Service users' } \\
\text { perspectives } \\
\text { towards } \\
\text { discrimination } \\
\text { and } \\
\text { interventions } \\
\text { to reduce } \\
\text { stigma } \\
\text { against } \\
\text { people with } \\
\text { mental illness }\end{array}$ & $\begin{array}{l}\text { Nearly less than one third of } \\
\text { participants had received the minit } \\
\text { required treatment available at dis } \\
\text { level. The follow up has revealed t } \\
\text { those who received the treatment } \\
\text { significant improvement in severe } \\
\text { mental illness symptoms, disabilit } \\
\text { measures, depression symptoms, } \\
\text { discrimination, restraining, alcohol } \\
\text { disorder and suicidal attempt }\end{array}$ \\
\hline $\begin{array}{l}\text { Asher, L., et al. } \\
\text { (2021). (Lancet } \\
\text { preprint) }\end{array}$ & $\begin{array}{l}\text { Quantitative } \\
\text { study } \\
\text { (Cluster- } \\
\text { randomized } \\
\text { controlled } \\
\text { trial) }\end{array}$ & $\begin{array}{l}\text { In intervention and } \\
\text { control arm, } 79 \text { and } 87 \\
\text { participants with } \\
\text { schizophrenia } \\
\text { respectively }\end{array}$ & $\begin{array}{l}\text { Community } \\
\text { based study at } \\
\text { Sodo district, } \\
\text { rural South- } \\
\text { central } \\
\text { Ethiopia }\end{array}$ & $\begin{array}{l}\text { WHO Disability } \\
\text { Assessment } \\
\text { Schedule } \\
\text { (WHODAS 2·0), } \\
\text { Brief Psychiatric } \\
\text { Rating Scale- } \\
\text { Expanded } \\
\text { (BPRSE) score, } \\
\text { Clinical Global } \\
\text { Impression } \\
\text { (CGI), Butajira } \\
\text { Functioning } \\
\text { Scale score, } \\
\text { Chart Schedule, } \\
\text { Adapted Client } \\
\text { Service Receipt } \\
\text { Inventory (CSRI), } \\
\text { Discrimination } \\
\text { and Stigma } \\
\text { Scale-12 (DISC- } \\
\text { 12), Involvement } \\
\text { Evaluation } \\
\text { Questionnaire } \\
\text { (IEQ) and } \\
\text { Patient Health } \\
\text { Questionnaire-9 } \\
\text { (PHQ-9) }\end{array}$ & $\begin{array}{l}\text { Service users' } \\
\text { perspectives } \\
\text { towards } \\
\text { interventions } \\
\text { to reduce } \\
\text { stigma } \\
\text { against } \\
\text { people with } \\
\text { mental illness }\end{array}$ & $\begin{array}{l}\text { After } 12 \text { months of community ba: } \\
\text { rehabilitation intervention, disabilit } \\
\text { was effectively reduced. However, } \\
\text { didn't found evidence on reduction } \\
\text { stigma. }\end{array}$ \\
\hline
\end{tabular}

\section{Explanatory models of stigma}

\section{Mental illness labels}

An experience shared by an Ethiopian living abroad and on a temporary visit in the country, stated that she heard the terms "insane" and "crazy" rather than "mentally ill" when national media made announcements for missing people with mental illness [36]. Similarly a clinical psychologist claimed that her clients were repeatedly using the term "I must be really crazy" [37].

In a qualitative study among mental health providers, most respondents perceived mental health as a basic component of a person's wellbeing. Some of the participants reported mental health as inter-connectedness of psychological, physical and emotional wellbeing. Others described mental health as being free from mental illness or ability to perform daily functions, and a similar number of respondents described it in relation to behaving in an acceptable way in a society or workplace [38].

\section{Perceived causes and symptoms of mental illnesses}

In a focus group discussion among religious leaders, health workers and community participants in the northern part of the country, the perceived common causes of mental illness pointed out were; controlled by evil spirit due to violation of God's rules, attack by devil spirit, grief from loss of a loved one, poverty, too much thinking, and substance use [39]. In the same study, most participants characterized people with mental illness as aggressive and violent physically. They also mentioned the fear of danger imposed by people with mental illness as a cause of stigma. In a study among health workers, community members and traditional healers, participants perceived that the expression of negative emotions could help to recognize depression and anxiety, whereas bizarre or unusual behavior could help to recognize psychosis [40]. 
A study in a rural part of the country revealed gender-related differences. Community responses to individuals with severe mental illness were reported to vary according to gender and visible symptoms in public spaces. Participants described feelings of sympathy and pity towards women who were visibly ill in public spaces. The reason for being more compassionate was due to considering the impact of the illness on a woman's domestic role, and the ability to have and care for her family. According to this study, diagnoses of mental illness in women were often kept secret or concealed within the home. Women with mental illnesses were still responsible for their family and household obligations and received less social support from their family. This made women vulnerable to neglect, sexual exploitation, and abuse [41].

According to one study among health workers, community members and traditional healers, the perceived causes of mental illnesses were related to psychocultural inappropriateness (culturally unacceptable behaviors, violating societal taboos, unfavorable attitudes, and lack of balance towards socially acceptable values), religious/spiritual factors, social difficulty, behavioral disturbance, cognitive-emotional impairment, disaster and economic deprivation, difficulties in adapting to environmental changes, substance abuse, and physical/medical conditions [40]. In a population-level study of mental distress in a rural part of the country, psychosocial stressors/stressful life events were predominant and strongly related with common mental illnesses [42].

\section{Perceived and experienced forms of stigma}

\section{Public stigma}

According to a qualitative study which involved 26 mental health practitioners or educators, participants perceived community stigma as soon as individuals with severe mental illness were seen outside their homes, regardless of gender [38]. In a population-based study among people with severe mental illness, $63.3 \%$ of 300 participants had experienced discrimination in the previous year, mainly by being avoided or shunned because of mental illness [43]. An institution-based study in an urban setting reported similar findings of perceived stigma (62.6\%) among 423 people with schizophrenia [44].

A study that assessed the community's attitude towards mentally ill in the south-west part of Ethiopia has shown that rural residents had significantly higher scores for perceived stigma as compared to urban residents. It was indicated that there was a negative association between degree of perceived stigma and the level of education. Those with higher scores of perceived supernatural causes or perceived psychosocial and biological causes had lower stigma scores [45]. In another similar study in the general public, there were higher perceived stigma scores towards people with mental illness among those with government or private employment, who were married, or had completed secondary school as compared to housewife, single, or completed primary education respectively [46].

In a facility-based study of south-west Ethiopia, the prevalence of perceived high and low stigma was $51 \%$ and $44 \%$ respectively among 384 people with mental illness seeking outpatient mental health care. In this study, substance use history, lack of family support and medication side effects were associated with higher perceived stigma [47]. In a qualitative study in a rural region of Ethiopia, the chance of marrying was perceived to be lower for individuals (both genders) with severe mental illness, because of the difficulty in finding a partner and starting a family due to perceived dangerousness and the prevalent stigma of mental illness. Men had a better chance of finding a partner after treatment than women, as impaired functioning due to severe mental illness was perceived to affect the ability to cook, care and clean among women. Women also have a higher risk of being separated or divorced due to a mental illness. If a woman was unable to perform the expected family role, the participants mentioned that she might be sent back to her family. For men, the risk of being abandoned due to mental illness was much lower [48].

\section{Structural stigma}

There are only few studies assessing structural stigma in Ethiopia. One of these is a qualitative study that included representatives from national and regional planners, service developers and policy makers of Ethiopia. The participants mentioned that there was government commitment and support for integration of mental health services at the primary health care level. Nevertheless, it was described that there were problems with regards to awareness, transparency, stakeholder involvement and coordination in mental health care planning and decision making. In addition, shortage of medication supplies and lack of community mobilization for mental health, and inadequate health management information system indicators needed for monitoring were among the structural barriers for mental health care scale-up in Ethiopia [49]. In a related community-based study among people with severe mental illness, those who were never engaged with mental health care perceived the cost of treatment as one of the barriers, and the fear of being treated differently was also mentioned by some participants [50].

In a qualitative study involving service users, caregivers and service providers, it was mentioned that the longer duration of treatment and inaccessible nature of the facilities providing the care were identified as the main barriers for engagement with mental health care, in addition to financial problems [51]. Additionally, people with schizophrenia and their caregivers had limited capacity to make a decision as the treatment options were limited, not feasible and difficult to access [52].

In a study about barriers and facilitators of service user involvement in mental health system strengthening, participants perceived prevailing negative attitudes towards mental health and towards people with mental illness among service providers, managers and policy developers. Lack of prioritization of mental health in policy development, and a lack of legislation, or culture of working with people with mental illness as partners were additional structural barriers for involvement of service users in policy drafting and planning, service development and quality monitoring [53]. A similar qualitative study on service user involvement in mental health system strengthening found unreliable medication supplies as a source of dissatisfaction among service users and service providers. Additionally, problems with regards to communication between service users and providers and a lack of evidence-based information about the medication options were also mentioned as barriers for involvement [54].

\section{Courtesy stigma}


Community-based studies in the south-west part of Ethiopia indicated that caregivers of people with mental illness isolated themselves from social life due to fear of public stigma and discrimination $[55,56]$. They felt shame or embarrassment about the family member's illness. They were worried that other people would find out about the illness and preferred to hide and keep it as a secret from other people and when visiting social events with their family member. It was indicated that family stigma was found to be moderately high and that being a rural resident was significantly associated with higher stigma scores. However, having a better explanation of mental illnesses was associated with decreased stigma.

\section{Self-stigma}

A study in a specialized psychiatric hospital in Addis Ababa indicated internalized stigma as the prevailing problem among people with schizophrenia. Nearly half of 212 participants had moderate to high self-stigma scores [57]. In another study from the same setting, around half of the participants reported low stigma resistance (the ability to remain unaffected by the stigma) [58].

An investigation from southern rural Ethiopia indicated that more than two thirds of people with alcohol use disorder had internalized stigma [59]. In a similar study, higher self-stigma scores were related to being female, having a history of traditional treatment, and a higher perceived supernatural explanation of mental illness [60].

\section{Impact of stigma on help-seeking}

There can be delayed treatment seeking behavior among people with mental illness. In a study among people with alcohol use disorder in a rural south-central part of Ethiopia, more than $80 \%$ of the participants did not receive any medical help for their illness [59]. Another study in south-west Ethiopia has showed that $65.1 \%$ of participants came for treatment after significant delays and more than half of them had tried traditional options (religious or herbal) before their arrival to the health facility. Those with symptoms like abdominal pain and headache were more likely to seek care early [61].

According to a qualitative study among mental healthcare providers [38], the majority of participants perceived that people should rely mainly on interventions from religious institutions to address mental health and wellbeing issues. This factor, in addition to inaccessibility of mental healthcare, is related to the high stigma around seeking modern mental health interventions, as opposed to the normative process of seeking help from the church. In another communitybased study on plans to improve mental health care access among people with severe mental illness, some respondents perceived stigma to be one of the barriers to non-engagement, although they did not think it would prevent attendance for most people [50].

The practice of involving people with mental illnesses in decision making on treatment options is not common. A study in a rural part of the country among people with schizophrenia revealed that the main decision makers were the caregivers and health workers. People with mental illnesses were merely consulted with regard to their treatment options, which indicated the prevailing pervasive stigma.

A study in a rural setting of Ethiopia found that people with severe mental illness were more likely to be victims than perpetrators of violence, but that perpetration of violence was more common than in the general population and reflected a lack of access to mental health care [62]. A study in a rural central part of the country among people with psychosis showed that nearly half had lifetime or current gaps in accessing biomedical care, and from those who received care, more than two thirds had minimally inadequate care [63].

\section{Interventions to reduce stigma}

A research PRogramme for Improving Mental HealthcarE (PRIME), which evaluated the impact of integrated mental health care in primary care in rural Ethiopia highlighted that, in addition to enhancing mental health care access and work functioning of people with severe mental illness, reducing discrimination could be important to reduce household food insecurity [64]. In another report of the same programme, although only less than one third of participants had received the minimum required treatment available at district level, there were significant improvements in clinical and social outcomes of people with severe mental illness. It was revealed that those who received the treatment had significant improvement in severe mental illness symptoms, disability measures, depression symptoms, discrimination, restraint, alcohol use disorder and suicidal attempt [65].

Studies conducted to develop and evaluate the community-based rehabilitation intervention (CBR) for people with schizophrenia in Ethiopia (RISE) have indicated that the approach could be cost-effective and acceptable, if it could be used with the available care in the facility and considering the setting of the community. This intervention approach is composed of home visits by trained (about the intervention) representatives from the community, community mobilization, and family support groups $[66,67]$. As per findings of 12-month cluster randomized controlled trial from RISE, disability was effectively reduced following a combination of CBR and task-shared facility-based care for people with schizophrenia. However, there was no evidence that CBR had an impact on discrimination as measured by Discrimination and Stigma Scale-12 (DISC-12) [68].

In research to inform the development of care for people with severe mental illness, stakeholders mentioned that the interventions should focus on reestablishing the living conditions of people with severe mental illness. They described that it could be challenging and might need a longer duration to achieve daily necessities, as poverty is the underlying condition for them. The innovative care for chronic conditions framework was adapted for the rural setting considering the importance of tackling stigma in the community, and using the role and resources of the families, community workers, traditional and religious leaders in addition to biomedical care [69].

It was indicated that awareness-raising component was included as a strategy for reduction of stigma and discrimination at the community level in rural Ethiopia [70]. Thus, using the community health workers especially health extension workers in rural Ethiopia could be important to enhance the community awareness due to their proximity to the community. Engaging the service users and caregivers in the awareness raising training could be effective in reducing public stigma [54]. 
An assessment of health workers' beliefs and attitudes in rural Ethiopia showed that after awareness enhancing training was provided to health workers on mental and developmental disorders, the level of stigmatizing attitudes decreased as indicated by lower scores on the social distance scale [71]. In one study it was suggested that empowerment with psychosocial interventions and addressing drug side effects could be helpful in reducing self-stigma among people with mental illnesses [60]. Another qualitative study in a rural setting has suggested that in addition to enhancing the access to mental health care, community outreach and mental health awareness raising programs could be an approach to reduce stigma and improve social outcomes of people with severe mental illness [48]. Similarly, interventions targeting the prevention of internalized stigma could reduce the functional impairment of people with severe mental illness [72].

\section{Discussion}

In our evidence synthesis we found that, in Ethiopia, people with mental illnesses were exposed to prevailing public stigma [38, 43-47, 51, 53], structural stigma $[49,50,53,54,73]$, courtesy stigma $[55,56]$ and self-stigma [57-60]. The fear of danger from aggressive and violent behavior was claimed as a reason for the stigma [39], which was also a reason mentioned in Asia [74]. Even though it is common to show sympathy for women with mental illnesses, it could be very challenging for them to attain their normal life [41], especially in finding a partner and establishing a family [48]. Additionally, there was low stigma resistance [58] and delayed treatment seeking [61] for mental illnesses, as it was common to believe in divine and traditional healing systems [38-40].

We did not find any intervention studies with the main aim of reducing stigma and discrimination in our setting. However, there were two studies, the RISE [68] and PRIME [65] studies, which had secondary outcomes of stigma reduction. The RISE study did not find evidence of reduction of discrimination by community-based rehabilitation. However, the PRIME results showed reduced discrimination, that is, increased access to effective mental health care. Thus, the following suggestions were also provided based on the findings of other studies with different primary objectives. In order to reduce stigma, economic empowerment [48] was suggested, as poverty was claimed to be a cause of stigma in Africa [21]. There were also initiatives underway to use social contact interventions [54]. However, awareness raising $[45,48,60]$ was the main intervention recommended. This might be the appropriate approach, as evidence [7, 17] supports education as an effective approach to address mental illness stigma. Although outcomes were not available at the time of this review, stigma reduction among primary care workers through social contact with service has been piloted in Ethiopia, based on the REducing Stigma among HealthcAre providErs (RESHAPE) intervention developed in Nepal [75]. As was stated in the vision of the WHO mental health strategy [4], people with mental illness should attain a full range of health and social services free from stigma and discrimination. However, achieving this might be challenging in Ethiopia, as mental illness was not accepted as culturally appropriate [21] and the terms used to describe the problem [36, 37] were stigmatizing. Besides, evidence [76] has indicated that all countries could fail to achieve the universal health coverage (UHC) goal for mental illnesses, if they were to continue with systems having stigmatizing barriers in relation to mental health services budgets.

The WHO has emphasized that mental health must be an integral part of UHC with adequate and quality services [2]. However, inaccessible mental health care services especially for people who are living in rural areas of the country is still a challenge [62, 77]. Another health system factor which contributes to prevailing stigma in the country is related to mental health workforce and awareness problems. Ethiopia has five times less mental health workforce staff than the global average, which places the country even below the average of low-income countries [78-80] and WHO recommendations of access to mental health care $[4,77]$. In addition, there are gaps in involving service users in mental health care - they have limited opportunities to make a decision on treatment options [52].

Stigma is pervasive and affects communities, healthcare providers, planners and policy-makers. There is low mental health awareness among service users [81], service providers [82, 83] and policy makers [49], accompanied by under-investment in mental health care [84] which might further contribute to the prevailing structural stigma. Even though there are opportunities [84], it could be difficult for Ethiopia to achieve UHC goal for mental illnesses, as it is required to increase service coverage by $50 \%$ for severe mental health conditions [2]. It should be noted that, in addition to monitoring the implementation of the plans, if the capacity building activities [85] and context specific strategies [84] could be applied effectively, including reduction of structural stigma, there is still hope of achieving the UHC goal for mental health disorders.

There is limited availability of evidence-based mental health care in Ethiopia, resulting in a high treatment gap [63]. From an international review [86], stigma reduction interventions were known to improve the lives of people with mental illnesses as indicated by a multilevel model containing the framework of interventions to reduce excess mortality. Addressing mental health stigma could also be helpful to achieve UHC for mental illness as per the evidence from a global review [76]. Therefore, evidence generated from this synthesis could be used as an up-to-date summary of the available evidence regarding Ethiopia's mental health stigma context. However, we acknowledge that this evidence synthesis should be supported by nationally representative primary data.

\section{Conclusion}

This study showed the prevailing public, structural, courtesy forms of stigma and self-stigma in different parts of Ethiopia, which may adversely affect quality of life and help-seeking behaviour by people with mental illness. Achieving the UHC goal by 2030 may not be realistic in the country without integrating mental health stigma-reduction approaches into efforts to scale up mental health care. However, it can be possible to achieve the goal if context specific strategies are developed, implementation of the plans are monitored, the available opportunities used effectively, and if capacity building activities are enhanced.

Moreover, there should be engagement of mental health service stakeholders (service users, caregivers, religious institutions, community leaders, local social services, primary care providers, specialists and policy makers) in the process of designing or adapting stigma interventions, so that these could be contextually realistic, effective and sustainable.

\section{Declarations}




\section{Acknowledgements}

BAK is supported by the U.S. National Institute of Mental Health (R01MH120649). GT is supported by the National Institute for Health Research (NIHR) Applied Research Collaboration South London at King's College London NHS Foundation Trust. CH, AA, and GT are funded through the ASSET research programme, supported by the UK's National Institute of Health Research (NIHR) using Official Development Assistance (ODA) funding (NIHR Global Health Research Unit on Health Systems Strengthening in Sub-Saharan Africa at King's College London (16/136/54)). The views expressed in this publication are those of the authors and not necessarily those of the NHS, the National Institute for Health Research or the Department of Health and Social Care, England. CH also receives support from AMARI as part of the DELTAS Africa Initiative [DEL- [15-01]. GT is also supported by the Guy's and St Thomas' Charity for the On Trac project (EFT151101), and by the UK Medical Research Council (UKRI) in relation to the Emilia (MR/S001255/1) and Indigo Partnership (MR/R023697/1) awards. PCG is supported by the UK Medical Research Council in relation to the Indigo Partnership (MR/R023697/1) award.

Ethics approval and consent to participate: Not applicable

Consent for publication: Not applicable

Availability of data and materials: The datasets used and/or analyzed during the current study are available from the corresponding author on reasonable request.

Competing interests: The authors declare that they have no competing interests.

Funding: No funding was obtained for this study

Authors' contributions: EG, BK, TM, CH, BK, SW, EH, PG, GT designed the study, participated in the analysis, write-up and critical review of the manuscript. All authors read and approved the final manuscript.

\section{References}

1. James SL, Abate D, Abate KH, Abay SM, Abbafati C, Abbasi N, Abbastabar H, Abd-Allah F, Abdela J, Abdelalim A et al: Global, regional, and national incidence, prevalence, and years lived with disability for 354 diseases and injuries for 195 countries and territories, 1990-2017: a systematic analysis for the Global Burden of Disease Study 2017. Lancet (London, England) 2018, 392(10159):1789-1858.

2. World Health Organization.: The WHO special initiative for mental health (2019-2023): universal health coverage for mental health. In.: World Health Organization; 2019.

3. Thornicroft G, Brohan E, Rose D, Sartorius N, Leese M: Global pattern of experienced and anticipated discrimination against people with schizophrenia: a cross-sectional survey. Lancet (London, England) 2009, 373(9661):408-415.

4. World Health Organization.: Mental health action plan 2013-2020. 2013.

5. Semrau M, Evans-Lacko S, Koschorke M, Ashenafi L, Thornicroft G: Stigma and discrimination related to mental illness in low- and middle-income countries. Epidemiology and psychiatric sciences 2015, 24(5):382-394.

6. Pescosolido BA, Martin JK: The Stigma Complex. Annu Rev Socio/ 2015, 41:87-116.

7. Gronholm PC, Henderson C, Deb T, Thornicroft G: Interventions to reduce discrimination and stigma: the state of the art. Social psychiatry and psychiatric epidemiology 2017, 52(3):249-258.

8. Bitew T: Prevalence and risk factors of depression in Ethiopia: a review. Ethiopian journal of health sciences 2014, 24(2):161-169.

9. IHME.: GBD Compare: Ethiopia. 2019, http://www.healthdata.org/ethiopia.

10. Fekadu A, Medhin G, Kebede D, Alem A, Cleare AJ, Prince M, Hanlon C, Shibre T: Excess mortality in severe mental illness: 10-year population-based cohort study in rural Ethiopia. The British journal of psychiatry : the journal of mental science 2015, 206(4):289-296.

11. Teferra S, Shibre T, Fekadu A, Medhin G, Wakwoya A, Alem A, Kullgren G, Jacobsson L: Five-year mortality in a cohort of people with schizophrenia in Ethiopia. BMC Psychiatry 2011, 11(1):165.

12. Gureje O, Alem A: Mental health policy development in Africa. Bulletin of the world health organization 2000, 78:475-482.

13. Ayano G, Assefa D, Haile K, Bekana L: Experiences, strengths and challenges of integration of mental health into primary care in Ethiopia. Experiences of East African Country. Fam Med Med Sci Res 2016, 5(204):2.

14. Federal Democratic Republic of Ethiopia Ministry of Health.: Health Sector Transformation Plan 2015/16-2019/20 (2008-2012 EFY). In.: Federal Democratic Republic of Ethiopia Ministry of Health; 2015.

15. Corrigan PW, River LP, Lundin RK, Penn DL, Uphoff-Wasowski K, Campion J, Mathisen J, Gagnon C, Bergman M, Goldstein H et al: Three strategies for changing attributions about severe mental illness. Schizophrenia bulletin 2001, 27(2):187-195.

16. Committee on the Science of Changing Behavioral Health Social Norms; Board on Behavioral C, and Sensory Sciences; Division of Behavioral and Social Sciences and Education; National Academies of Sciences, Engineering, Medicine: Ending Discrimination Against People With Mental and Substance Use Disorders: The Evidence for Stigma Change, https://www.ncbi.nlm.nih.gov/books/NBK384914/. Washington (DC): National Academies Press (US); 2016. 
17. Morgan AJ, Reavley NJ, Ross A, Too LS, Jorm AF: Interventions to reduce stigma towards people with severe mental illness: Systematic review and meta-analysis. Journal of psychiatric research 2018, 103:120-133.

18. Corrigan PW, Morris SB, Michaels PJ, Rafacz JD, Rüsch N: Challenging the public stigma of mental illness: a meta-analysis of outcome studies. Psychiatric services (Washington, DC) 2012, 63(10):963-973.

19. Mehta N, Clement S, Marcus E, Stona AC, Bezborodovs N, Evans-Lacko S, Palacios J, Docherty M, Barley E, Rose D et al: Evidence for effective interventions to reduce mental health-related stigma and discrimination in the medium and long term: systematic review. The British journal of psychiatry : the journal of mental science 2015, 207(5):377-384.

20. Tanaka C, Tuliao MTR, Tanaka E, Yamashita T, Matsuo H: A qualitative study on the stigma experienced by people with mental health problems and epilepsy in the Philippines. BMC psychiatry 2018, 18(1):325.

21. Amuyunzu-Nyamongo M: The social and cultural aspects of mental health in African societies. Commonwealth health partnerships 2013, 2013:59-

63.

22. Thornicroft G, Bakolis I, Evans-Lacko S, Gronholm Petra C, Henderson C, Kohrt BA, Koschorke M, Milenova M, Semrau M, Votruba N et al: Key lessons learned from the INDIGO global network on mental health related stigma and discrimination. World psychiatry : official journal of the World Psychiatric Association (WPA) 2019, 18(2):229-230.

23. Brohan E, Clement S, Rose D, Sartorius N, Slade M, Thornicroft G: Development and psychometric evaluation of the Discrimination and Stigma Scale (DISC). Psychiatry research 2013, 208(1):33-40.

24. Lanfredi M, Zoppei S, Ferrari C, Bonetto C, Van Bortel T, Thornicroft G, Knifton L, Quinn N, Rossi G, Lasalvia A: Self-stigma as a mediator between social capital and empowerment among people with major depressive disorder in Europe: the ASPEN study. European psychiatry : the journal of the Association of European Psychiatrists 2015, 30(1):58-64.

25. Lasalvia A, Van Bortel T, Bonetto C, Jayaram G, van Weeghel J, Zoppei S, Knifton L, Quinn N, Wahlbeck K, Cristofalo D et al: Cross-national variations in reported discrimination among people treated for major depression worldwide: the ASPEN/INDIGO intemational study. The British journal of psychiatry : the journal of mental science 2015, 207(6):507-514.

26. Lasalvia A, Zoppei S, Van Bortel T, Bonetto C, Cristofalo D, Wahlbeck K, Bacle SV, Van Audenhove C, van Weeghel J, Reneses B et al: Global pattern of experienced and anticipated discrimination reported by people with major depressive disorder. a cross-sectional survey. Lancet (London, England) 2013 , 381(9860):55-62.

27. Quinn N, Knifton L, Goldie I, van Bortel T, Dowds J, Lasalvia A, Scheerder G, Boumans J, Svab V, Lanfredi M et al: Nature and impact of European antistigma depression programmes. Health promotion international 2014, 29(3):403-413.

28. Zoppei S, Lasalvia A, Bonetto C, Van Bortel T, Nyqvist F, Webber M, Aromaa E, Van Weeghel J, Lanfredi M, Harangozó J et al: Social capital and reported discrimination among people with depression in 15 European countries. Social psychiatry and psychiatric epidemiology 2014, 49(10):1589-1598.

29. Thornicroft G, Mehta N, Clement S, Evans-Lacko S, Doherty M, Rose D, Koschorke M, Shidhaye R, O'Reilly C, Henderson C: Evidence for effective interventions to reduce mental-health-related stigma and discrimination. Lancet (London, England) 2016, 387(10023):1123-1132.

30. Central Intelligence Agency.: The World Factbook: Ethiopia. 2020, https://www.cia.gov/library/publications/the-world-factbook/geos/et.html

31. Population EOot, Commission HC: Summary and statistical report of the 2007 population and housing census: population size by age and sex: Federal Democratic Republic of Ethiopia, Population Census Commission; 2008.

32. Health Policy of Transitional Government of Ethiopia.: Health Policy of the Transitional Government of Ethiopia. . 1993.

33. Federal Democratic Republic of Ethiopia Ministry of Health.: National Mental Health Strategy 2012/13-2015/16. In.: Ministry of Health Addis Ababa; 2012.

34. Ministry of Heath of th FDRE.: National Mental Health Strategy 2021-2025 2021, https://www.govserv.org/ET/Addis-

Ababa/241465976024213/Ministry-of-Health\%2CEthiopia.

35. World Health Organization and Ethiopian FMOH.: mhGAP in Ethiopia: Proof of Concept. In.,

https://www.mhinnovation.net/sites/default/files/downloads/resource/mhGap\%20in\%20Ethiopia-\%20Proof\%20of\%20Concept.pdf. Geneva; 2013.

36. Elezebet Mitiku.: My experiences with the mental health stigma in Ethiopia [https://threesixty.stthomas.edu/mental-illness-and-my-community-myexperiences-with-the-mental-health-stigma-in-ethiopia/]

37. Yeshashwork Kibour.: Mind the Gap: Personal Reflections on the Mental Health Infrastructure of Ethiopia. In., https://www.apa.org/international/pi/2010/04/reflections: American Psychological Association; 2010. 
39. Yeshanew B, Belete A, Necho M: Help-seeking intention and associated factors towards mental illness among residents of Mertule Mariam town, East Gojam Zone, Amhara Region, Ethiopia: a mixed-method study. Annals of general psychiatry 2020, 19(1):1-11.

40. Monteiro NM, Balogun SK: Perceptions of mental illness in Ethiopia: a profile of attitudes, beliefs and practices among community members, healthcare workers and traditional healers. International Journal of Culture and Mental Health 2014, 7(3):259-272.

41. Ghebrehiwet S, Baul T, Restivo JL, Kelkile TS, Stevenson A, Gelaye B, Fekadu A, Hailemariam M, Girma E, Teferra S: Gender-specific experiences of serious mental illness in rural Ethiopia: A qualitative study. Global public health 2019:1-15.

42. Fekadu A, Medhin G, Selamu M, Hailemariam M, Alem A, Giorgis TW, Breuer E, Lund C, Prince M, Hanlon C: Population level mental distress in rural Ethiopia. BMC psychiatry 2014, 14(1):194.

43. Forthal S, Fekadu A, Medhin G, Selamu M, Thornicroft G, Hanlon C: Rural vs urban residence and experience of discrimination among people with severe mental illnesses in Ethiopia. BMC psychiatry 2019, 19(1):340.

44. Tesfaw G, Kibru B, Ayano G: Prevalence and factors associated with higher levels of perceived stigma among people with schizophrenia Addis Ababa, Ethiopia. International Journal of Mental Health Systems 2020, 14(1):19.

45. Girma E, Tesfaye M, Froeschl G, Möller-Leimkühler AM, Müller N, Dehning S: Public stigma against people with mental illness in the Gilgel Gibe Field Research Center (GGFRC) in Southwest Ethiopia. PLoS One 2013, 8(12):e82116.

46. Reta Y, Tesfaye M, Girma E, Dehning S, Adorjan K: Public stigma against people with mental illness in Jimma Town, Southwest Ethiopia. PloS one 2016, 11(11).

47. Hadera E, Salelew E, Girma E, Dehning S, Adorjan K, Tesfaye M: Magnitude and Associated Factors of Perceived Stigma among Adults with Mental IIIness in Ethiopia. Psychiatry journal 2019, 2019.

48. Hailemariam M, Ghebrehiwet S, Baul T, Restivo JL, Shibre T, Henderson DC, Girma E, Fekadu A, Teferra S, Hanlon C: “He can send her to her parents”: The interaction between marriageability, gender and serious mental illness in rural Ethiopia. BMC psychiatry 2019, 19(1):315.

49. Hanlon C, Eshetu T, Alemayehu D, Fekadu A, Semrau M, Thornicroft G, Kigozi F, Marais DL, Petersen I, Alem A: Health system governance to support scale up of mental health care in Ethiopia: a qualitative study. Int J Ment Health Syst 2017, 11:38.

50. Hailemariam M, Fekadu A, Medhin G, Prince M, Hanlon C: Equitable access to mental healthcare integrated in primary care for people with severe mental disorders in rural Ethiopia: a community-based cross-sectional study. Int J Ment Health Syst 2019, 13:78.

51. Hailemariam M, Fekadu A, Prince M, Hanlon C: Engaging and staying engaged: a phenomenological study of barriers to equitable access to mental healthcare for people with severe mental disorders in a rural African setting. Int J Equity Health 2017, 16(1):156.

52. Souraya S, Hanlon C, Asher L: Involvement of people with schizophrenia in decision-making in rural Ethiopia: a qualitative study. Globalization and health 2018, 14(1):85.

53. Abayneh S, Lempp H, Alem A, Alemayehu D, Eshetu T, Lund C, Semrau M, Thornicroft G, Hanlon C: Service user involvement in mental health system strengthening in a rural African setting: qualitative study. BMC Psychiatry 2017, 17(1):187.

54. Abayneh S, Lempp H, Alem A, Kohrt BA, Fekadu A, Hanlon C: Developing a Theory of Change model of service user and caregiver involvement in mental health system strengthening in primary health care in rural Ethiopia. International Journal of Mental Health Systems 2020, 14(1):1-17.

55. Girma E, Möller-Leimkühler AM, Müller N, Dehning S, Froeschl G, Tesfaye M: Public stigma against family members of people with mental illness: findings from the Gilgel Gibe Field Research Center (GGFRC), Southwest Ethiopia. BMC international health and human rights 2014, 14:2.

56. Girma E, Möller-Leimkühler AM, Dehning S, Mueller N, Tesfaye M, Froeschl G: Self-stigma among caregivers of people with mental illness: toward caregivers' empowerment. Journal of multidisciplinary healthcare 2014, 7:37-43.

57. Assefa D, Shibre T, Asher L, Fekadu A: Internalized stigma among patients with schizophrenia in Ethiopia: a cross-sectional facility-based study. BMC psychiatry 2012, 12(1):239.

58. Bifftu BB, Dachew BA, Tiruneh BT: Stigma resistance among people with schizophrenia at Amanuel Mental Specialized Hospital Addis Ababa, Ethiopia: a cross-sectional institution based study. BMC Psychiatry 2014, 14:259.

59. Zewdu S, Hanlon C, Fekadu A, Medhin G, Teferra S: Treatment gap, help-seeking, stigma and magnitude of alcohol use disorder in rural Ethiopia Substance Abuse Treatment, Prevention, and Policy 2019, 14(1):4. 
60. Girma E, Tesfaye M, Froeschl G, Möller-Leimkühler AM, Dehning S, Müller N: Facility based cross-sectional study of self stigma among people with mental illness: towards patient empowerment approach. International journal of mental health systems 2013, 7(1):21.

61. Girma E, Tesfaye M: Patterns of treatment seeking behavior for mental illnesses in Southwest Ethiopia: a hospital based study. BMC Psychiatry 2011, 11(1):138

62. Tsigebrhan R, Shibre T, Medhin G, Fekadu A, Hanlon C: Violence and violent victimization in people with severe mental illness in a rural low-income country setting: a comparative cross-sectional community study. Schizophrenia research 2014, 152(1):275-282.

63. Fekadu A, Medhin G, Lund C, DeSilva M, Selamu M, Alem A, Asher L, Birhane R, Patel V, Hailemariam M et al: The psychosis treatment gap and its consequences in rural Ethiopia. BMC Psychiatry 2019, 19(1):325.

64. Tirfessa K, Lund C, Medhin G, Selamu M, Birhane R, Hailemichael Y, Fekadu A, Hanlon C: Impact of integrated mental health care on food insecurity of households of people with severe mental illness in a rural African district: a community-based, controlled before-after study. Tropical medicine \& international health : TM \& IH 2020, 25(4):414-423.

65. Hanlon C, Medhin G, Selamu M, Birhane R, Dewey M, Tirfessa K, Garman E, Asher L, Thornicroft G, Patel V et al: Impact of integrated district level mental health care on clinical and social outcomes of people with severe mental illness in rural Ethiopia: an intervention cohort study. Epidemiology and psychiatric sciences 2019, 29:e45.

66. Asher L, Hanlon C, Birhane R, Habtamu A, Eaton J, Weiss HA, Patel V, Fekadu A, De Silva M: Community-based rehabilitation intervention for people with schizophrenia in Ethiopia (RISE): a 12 month mixed methods pilot study. BMC Psychiatry 2018, 18(1):250.

67. Asher L, Fekadu A, Hanlon C, Mideksa G, Eaton J, Patel V, De Silva MJ: Development of a Community-Based Rehabilitation Intervention for People with Schizophrenia in Ethiopia. PLoS One 2015, 10(11):e0143572.

68. Asher L, Birhane R, Weiss HA, Medhin G, Selamu M, Patel V, De Silva M, Hanlon C, Fekadu A: Community-Based Rehabilitation Intervention for People with Schizophrenia in Ethiopia (RISE): Results of a 12-Month Cluster-Randomized Controlled Trial. Available at SSRN: https://ssrncom/abstract=3839407 or http://dxdoiorg/102139/ssrn38394072021.

69. Mall S, Hailemariam M, Selamu M, Fekadu A, Lund C, Patel V, Petersen I, Hanlon C: 'Restoring the person's life': a qualitative study to inform development of care for people with severe mental disorders in rural Ethiopia. Epidemiology and psychiatric sciences 2017, 26(1):43-52.

70. Fekadu A, Hanlon C, Medhin G, Alem A, Selamu M, Giorgis TW, Shibre T, Teferra S, Tegegn T, Breuer E et al: Development of a scalable mental healthcare plan for a rural district in Ethiopia. The British journal of psychiatry : the journal of mental science 2016, 208 Suppl 56(Suppl 56):s4-12.

71. Tilahun D, Fekadu A, Tekola B, Araya M, Roth I, Davey B, Hanlon C, Hoekstra RA: Ethiopian community health workers' beliefs and attitudes towards children with autism: Impact of a brief training intervention. Autism : the international journal of research and practice 2019, 23(1):39-49.

72. Habtamu K, Alem A, Medhin G, Fekadu A, Hanlon C: Functional impairment among people with severe and enduring mental disorder in rural Ethiopia: a cross-sectional study. Social psychiatry and psychiatric epidemiology 2018, 53(8):803-814.

73. Hailemariam M, Fekadu A, Selamu M, Medhin G, Prince M, Hanlon C: Equitable access to integrated primary mental healthcare for people with severe mental disorders in Ethiopia: a formative study. Int J Equity Health 2016, 15(1):121.

74. Zhang Z, Sun K, Jatchavala C, Koh J, Chia Y, Bose J, Li Z, Tan W, Wang S, Chu W: Overview of stigma against psychiatric illnesses and advancements of anti-stigma activities in six Asian societies. International journal of environmental research and public health 2020, $17(1): 280$.

75. Kohrt BA, Turner EL, Rai S, Bhardwaj A, Sikkema KJ, Adelekun A, Dhakal M, Luitel NP, Lund C, Patel V et al: Reducing mental illness stigma in healthcare settings: Proof of concept for a social contact intervention to address what matters most for primary care providers. Social science \& medicine (1982) 2020, 250:112852.

76. Patel V, Saxena S: Achieving universal health coverage for mental disorders. BMJ (Clinical research ed) 2019, 366:14516.

77. Federal Democratic Republic of Ethiopia Ministry of Health.: Mental Health for ARM. 2019, www.moh.gov.et > default > files > Mental Health for ARM 2011Recent.

78. World Health Organization.: Mental health atlas 2017. In. Geneva: World Health Organization; 2018.; 2018.

79. World Health Organization.: Mental Health Atlas 2017. Country Profiles. In., https://www.who.int/mental_health/evidence/atlas/profiles-2017/en. Geneva: WHO; 2018.

80. World Health Organization.: Global health observatory data repository. 2020. In: Geneva, Switzerland: World Health Organization. https://www.who.int/data/gho/data/themes/topics/indicator-groups/indicator-group-details/GHO; 2020.

81. Hanlon C, Luitel NP, Kathree T, Murhar V, Shrivasta S, Medhin G, Ssebunnya J, Fekadu A, Shidhaye R, Petersen I: Challenges and opportunities for implementing integrated mental health care: a district level situation analysis from five low-and middle-income countries. PloS one 2014, 9(2).

Page $17 / 18$ 
82. Ahmed E, Merga H, Alemseged F: Knowledge, attitude, and practice towards mental illness service provision and associated factors among health extension professionals in Addis Ababa, Ethiopia. International journal of mental health systems 2019, 13(1):5.

83. Girma S, Kitaw Y, Ye-Ebiy Y, Seyoum A, Desta H, Teklehaimanot A: Human resource development for health in Ethiopia: challenges of achieving the millennium development goals. The Ethiopian Journal of Health Development (EJHD) 2007, 21(3).

84. Hanlon C, Alem A, Lund C, Hailemariam D, Assefa E, Giorgis TW, Chisholm D: Moving towards universal health coverage for mental disorders in Ethiopia. International journal of mental health systems 2019, 13(1):11.

85. Semrau M, Alem A, Abdulmalik J, Docrat S, Evans-Lacko S, Gureje O, Kigozi F, Lempp H, Lund C, Petersen I: Developing capacity-building activities for mental health system strengthening in low-and middle-income countries for service users and caregivers, service planners, and researchers. Epidemiology and psychiatric sciences 2018, 27(1):11-21.

86. Liu NH, Daumit GL, Dua T, Aquila R, Charlson F, Cuijpers P, Druss B, Dudek K, Freeman M, Fujii C et al: Excess mortality in persons with severe mental disorders: a multilevel intervention framework and priorities for clinical practice, policy and research agendas. World psychiatry : official journal of the World Psychiatric Association (WPA) 2017, 16(1):30-40.

\section{Figures}

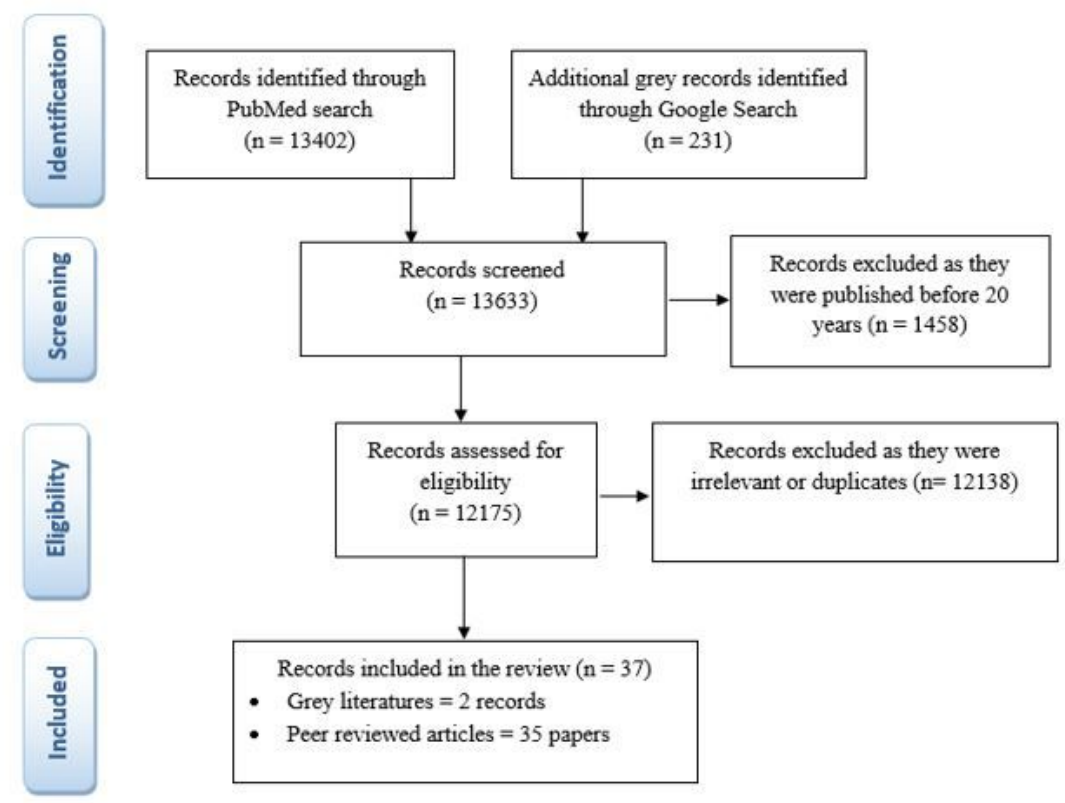

Figure 1

The PRISMA flow diagram

\section{Supplementary Files}

This is a list of supplementary files associated with this preprint. Click to download.

- Appendix1.docx 